\title{
Valoración del Paciente Suicida. Riesgos y Prevención.
}

\author{
Imanol Querejeta Ayerdi \\ Hospital Donostia. San Sebastián. \\ Universidad del País Vasco, Departamento de Neurociencias.
}

\section{Introducción}

La conducta suicida es una forma de comportamiento sustentada por motivaciones muy especiales. Su estudio resulta tan difícil y controvertido como extraer sus factores determinantes.

A pesar de que para muchos autores no está del todo claro, el suicidio es un acto y no se ajusta al modelo tradicional de enfermedad. En sí mismo, tampoco es una causa de muerte, ni un comportamiento que afecte sólo a personas enfermas. Las impresionantes imágenes de las personas lanzándose al vacío desde las torres del World Trade Centre de Nueva York tras el ataque aéreo del pasado II de septiembre de 200I, ilustran de forma dramática que la desesperación es una causa, probablemente la más frecuente, de esta forma de comportamiento.

Las fuentes de la O.M.S., que estiman que para el año 2020 aproximadamente I millón y medio de personas morirán como consecuencia de una tentativa de suicidio. También se estima que el número de personas que harán una tentativa sin resultado final de muerte (I) será 10 veces mayor. Esta información nos viene a decir que, en esas fechas, en el mundo habrá una muerte por suicidio cada 20 segundos y una tentativa cada $\mathrm{I}-2$ segundos.
En nuestro país el estudio de esta forma de comportamiento ha chocado contra tres importantes obstáculos. El primero la falta, hasta hace poco tiempo, de un procedimiento que investigue hasta los últimos detalles todas las muertes que se dan en circunstancias extrañas, muchas veces acompañadas de violencia y que han sido registradas como accidentes durante muchos años. En segundo lugar, el rechazo que siempre ha producido este tipo de conducta. En Europa en el siglo XIII se prohibía enterrar a las víctimas de suicidio en campo santo y sólo a principios del siglo XIX los ingleses abandonaron la costumbre de enterrar a los suicidas en los cruces de caminos (2). Por último, la necesidad que se ha tenido, por razones no siempre entendidas, de buscar una definición exacta de lo que es esta forma de conducta. En realidad en lo que hay una diferencia sustancial es entre los pacientes que hacen una tentativa de suicidio y los que consuman un suicidio, se puede aceptar que las tentativas de suicidio son conductas que buscan y consiguen la solución de un problema externo (3).

\section{FACTORES QUE INFLUYEN EN EL COMPORTAMIENTO SUICIDA}

Un factor de riesgo es un concepto estadístico y se define como la asociación entre 
alguna característica o atributo del individuo, grupo, o medio ambiente y el aumento de la probabilidad de que ocurra una enfermedad particular o un fenómeno relacionado con la enfermedad o problema objeto de estudio.

Teóricamente, los factores de riesgo ofrecen pistas sobre la causalidad de la enfermedad. Por ello los clínicos pueden usar los factores de riesgo para identificar a pacientes, determinar los períodos críticos de su enfermedad e instaurar una intervención médica adecuada (3).

Los factores de riesgo asociados a la conducta suicida son conocidos ya que fueron descritos por Durkheim en 1897 (4). Desde entonces hasta nuestros días ha variado la importancia de cada uno de ellos.

Distinguimos dos tipos de factores: Factores Socioambientales y Factores Individuales.

PERFIL DE LAS PERSONAS QUE COMETEN SUICIDIO COMPLETO

Varones (3 veces más que mujeres)

- Ancianos y adultos.

- Personas que utilizan métodos agresivos.

- Raza blanca

- Personas que viven solas

- Desempleados

- Clase social baja.

- Abuso de drogas.

- Enfermedades crónicas

- Antecedentes previos (3 veces más riesgo)

El aumento de las tasas de suicidio consumado en los ancianos se justifica porque a esa edad las personas están sometidas a cambios bruscos en su vida, disminución de su calidad de vida y con una disminución de recursos de todo tipo para hacer frente a situaciones inesperadas $(5,6)$.

En relación con el sexo, la mayoría de los autores coinciden en que los suicidios completos son más frecuentes en varones y los suicidios incompletos en mujeres $(7,8)$.
Otro factor relacionado con este tipo de conducta es el desempleo, sin que se llegue a saber qué es más importante, si la pérdida de la capacidad adquisitiva y el miedo al futuro asociado a esta situación $(9,10,11,12,13)$, o la pérdida de la autoestima, el contacto con los compañeros y el cambio de rol social. Esta asociación ha sido estudiada por varios autores que coinciden en que las tasas se multiplican hasta por 10 en el caso del suicidio no consumado tanto para los varones como para las mujeres. Todos estos estudios coinciden también en que el riesgo es más alto cuanto más largo es el período de desempleo.

\section{PERFIL DE LAS PERSONAS CON CONDUCTA SUICIDA INCOMPLETA}

- La proporción entre mujeres es 4 veces mayor

- Personas jóvenes (Menos recursos de afrontamiento)

- Método más usado $\rightarrow$ Medicación + Alcohol

- Personas que viven solas

- Desempleados

- Diagnóstico psiquiátrico

- Bajo nivel social. Abuso de drogas

- Trastornos de personalidad

\section{LA VALORACIÓN DEL RIESGO DE SUICIDIO COMO FORMA DE PREVENCIÓN}

Para los clínicos la mejor manera de hacer prevención es hacer una valoración, lo más exacta posible, de las personas que se atienden presentando un riesgo. Como la única herramienta de la que se dispone es la entrevista, explicaremos la información relevante que hay que obtener $y$ tener en cuenta para poder tomar decisiones correctas. La aplicación debe de ser rigurosa.

Para empezar, hay que distinguir el paciente que acude tras una tentativa de suicidio, del que acude a nuestra consulta sin adoptar esta forma de comportamiento. 
Se debe de prestar atención a los factores de riesgo a los que hemos hecho referencia porque cerca de la mitad de las personas que tienen una conducta suicida, completa o incompleta, han contactado con su médico de atención primaria en el período de ocho semanas anterior al acto suicida y lo hacen expresando sentimientos de hastío, desesperanza y haciendo mención al suicidio de forma más o menos explícita (14). Los autores citados refieren que de ese $48 \%$ de personas que han visitado a su médico de familia con anterioridad a un acto, el $31 \%$ de los que consuman el suicidio y el $20 \%$ de los que no lo consiguen han expresado ideas de muerte o falta de expectativas de futuro con anterioridad.

Una vez que sospechamos que un paciente sin antecedentes de este tipo de comportamiento que acude a nuestra consulta presenta un perfil de riesgo, debemos de tener presente que la probabilidad de cometer un suicidio aumenta de forma progresiva y continua en función del número de factores de riesgo que presente el paciente. Inicialmente el paciente afectado por algunos de los factores de riesgo ya comentado empieza a experimentar ideación vaga de suicidio. $A$ medida que pasa el tiempo sin que esta persona reciba el tratamiento adecuado, estas ideas difusas se empiezan a concretar y a convertirse en planes. En este momento debemos de abordar de una forma clara, explícita y sensible los problemas de nuestro paciente de riesgo. Si, en ausencia del efecto desinhibidor de una droga, normalmente el alcohol, una persona nos habla de planes concretos de suicidio debemos concederle el máximo riesgo y actuar para protegerle.

Cuando el paciente ya tiene una historia previa de un suicidio incompleto el riesgo es bastante mayor ya que la repetición es una de las variables que más frecuentemente se asocia al suicidio. De hecho, un $40 \%$ de las personas que completan un suicidio tienen tentativas previas. Diferentes estudios coinciden en que las personas que repiten después de un primer inten- to lo hacen en el período de 12 meses posterior al mismo $(15,16)$.

Cuando lo que tenemos que hacer es valorar un suicidio incompleto, sea en primera tentativa o sucesivas, es útil sistematizar la exploración con una entrevista rigurosa que se debería de completar con la aplicación de una escala específica de evaluación de este tipo de conducta.

La primera medida que hay que tomar cuando se va a evaluar a una persona por un suicidio incompleto es descartar de forma absoluta la existencia de alguna complicación médica y tratarla. A continuación se debe de proceder a una valoración psiquiátrica, que siempre que se pueda la hará un especialista.

Después hay que valorar la tentativa en si misma. Para ello el terapeuta que haga la valoración del suicidio incompleto debe de encontrar una respuesta a las siguientes preguntas:

I. ¿Cómo se explica la tentativa tanto en objetivos, como en razones?

2. ¿Cuál ha sido la gravedad del intento de suicidio?

3. ¿Persiste el riesgo de una tentativa o suicidio consumado en este momento?

4. ¿Qué problemas afectan al paciente? (Tanto los remotos como los inmediatos).

5. ¿Hay alguna situación que ha actuado como precipitante?

6. ¿El paciente evaluado padece una enfermedad psiquiátrica? ¿Cuál es el diagnóstico y la importancia en la tentativa de suicidio?

7. ¿Qué precisa el individuo evaluado? ¿Está capacitado para aprovechar esa ayuda?

El primer punto es el más importante de toda la entrevista y seguramente el más difícil. Hay que explorar las circunstancias y las expectativas que rodean la tentativa. También el por qué se da esta conducta en el momento que ocurre y no antes (esto de manera especial cuando una persona adopta esta forma de conducta tras muchos años de vivir con dificultades). Hay 
que saber si lo que el individuo espera conseguir con su conducta es la muerte.

El segundo punto a explorar es la intencionalidad real y para ello hay que explorar los siguientes puntos:

I. El intento es meditado durante tiempo y se hace acopio de material para llevar a cabo el acto. (Impulsivo o Planificado)

2. El individuo actúa buscando la soledad.

3. La intervención de alguna persona que le auxiliase era poco probable.

4. El individuo tomó precauciones para no ser descubierto.

5. El método utilizado. ¿Por qué ese y no otro?

6. Seguridad acerca de la letalidad del método elegido.

7. En los casos de sobreingesta de fármacos, el individuo se toma todos los disponibles.

8. El paciente no sólo no pide ayuda sino que se opone a ser auxiliado.

9. La presencia de actos finales como notas explicativas o últimas voluntades.

10. La admisión de una intencionalidad de muerte.

Cuantos más factores de los mencionados se detectan en la entrevista mayor es el riesgo

También es útil conocer el perfil que más frecuentemente se encuentra entre las personas que consuman un suicidio después de un intento fallido. Los más importantes de estos rasgos son los siguientes:

I. Mayor de 45 años.

2. Varón.

3. Desempleado.

4. Separado, divorciado o viudo.

5. Vivir sin acompañantes.

6. Mala salud física.

7. Llevar 6 meses en tratamiento médico.

8. Padecer alguna enfermedad psiquiátrica.

9. Abusar del alcohol u otras drogas.

10. Haber utilizado un método violento y potencialmente peligroso en la tentativa que se está valorando.
II. Presencia de una nota de despedida.

12. Tentativas previas.

Entre 2 y 5 factores la tasa de suicidio es $6.98 \times 1000$.

Entre los que cubren 10 ó más factores la tasa de suicidio es de $60 \times 1000$.

Estos factores fueron propuestos por Tuckman y Youngman (17) después de estudiar una muestra de 3800 personas con intentos de suicidio que posteriormente completaron una conducta suicida. Ellos encontraron que entre las personas entrevistadas que acumulaban de 2 a 5 de estos factores el riesgo de suicidio era de 6.98 por 1000 , mientras que en los que acumulaban entre 10 o más tenían una tasa de suicidio nueve veces mayor (60 por 1000).

En tercer lugar hay que conocer si el paciente está en riesgo de repetir su conducta, es decir, si en caso de tener un deseo claro de muerte éste persiste durante la entrevista. Si es así, se debe de contemplar la necesidad de una hospitalización involuntaria.

En cuarto lugar hay que distinguir los problemas que tiene para lo cual es aconsejable entrevistarse con los familiares más próximos del paciente.

Por último, una vez valorada toda esta información hay que decidir la ayuda que se ofrece al paciente y valorar si la va a aceptar. Cuando la indicación que se da contempla seguir un tratamiento ambulatorio, es aconsejable facilitar lo más posible el que el paciente salga de la entrevista con una cita y asegurarse, también, de que acude a la misma.

Toda esta información debe de obtenerse a través de un lenguaje claro y directo. La actitud debe de ser correcta, receptiva y guiada por el tacto y el interés hacia el paciente. De esta manera el paciente podrá expresar sentimientos y discutir acerca de sus problemas con una 
intensidad que antes no había hecho. No hay que olvidar que muchas personas que cometen un suicidio incompleto no son enfermos y que no van a requerir, ni desear, un seguimiento convirtiéndose esa entrevista en el único contacto que van a mantener con una persona entrenada en el manejo de esta situación.

Por último, es preciso que la entrevista se lleve a cabo tan pronto como el paciente esté en condiciones de atender a una entrevista. Si la valoración se hace cuando el paciente está afectado por las consecuencias físicas de su conducta (p.ej en el caso de intoxicación medicamentosa) la información puede ser imprecisa y la valoración incorrecta. Si, por el contrario ha pasado mucho tiempo después de la tentativa fallida, es muy probable que la situación esté parcialmente, que no bien, elaborada no pudiéndose llegar a conclusiones acertadas.

Para terminar y aunque la valoración de un acto suicida se debe de basar en una entrevista clínica.

\section{Sintetizando:}

- El principal problema que ha habido hasta ahora para el estudio de la conducta suicida es la existencia de discrepancias acerca de su definición.

- Al igual que en otras formas de comportamiento como el alcoholismo, parece más importante tener un concepto de lo que es la conducta suicida que hacer una definición exacta.

El factor de riesgo inmediato para que una persona consume un suicidio es la desesperación ante la falta de expectativas de futuro.

$>$ El factor de riesgo diferido más importante en el suicidio es el haber hecho tentativas previas, sobre todo en los 12 meses anteriores al acto definitivo.

> Para prevenir el suicidio hay que explorar la existencia de ideación autolítica cada vez que se atiende de urgencia a un paciente psiquiátrico.

En las personas con antecedentes, la exploración de las tentativas previas permite conocer su gravedad y el riesgo de repetición.

$>$ Los cuestionarios estandarizados son de utilidad en la valoración de este riesgo. En concreto los cuestionarios de Beck tiene una alta validez y utilidad.

> La hospitalización breve, si es preciso de forma involuntaria, contribuye a disminuir la tensión del paciente y acompañantes durante el tiempo que dura la exploración. También facilita el inicio de un seguimiento.

- Siempre que se entrevista a un paciente que presenta una conducta suicida hay que procurar, por todos los medios, entrevistar también a la familia y/o acompañantes en el momento de la acción.

Al hacer la exploración hay que valorar siempre todos los factores de mayor riesgo asociados a esta forma de conducta.

\section{BIBLIOGRAFÍA}

I. World Health Organization. Figures and facts about suicide (Doc. WHO/MNH/MDB/ 99.I) WHO: Geneva. 1999.

2. Rosen, G. History in the study of suicide. Psychological Medicine. I97I; I: 267-285.

3. Baechler, J. Suicide. Oxford: Basil Blackwell, 1980.

4. Berrios, $\mathbf{G}$ y Mohanna, M. Durkheim and french psychiatry views on suicidal during the I9th century. A conceptual history. British Journal of Psychiatry. 1990; 156: I-9.

5. Shah, AK., De,T. Suicide and the elderly. International Journal of Psychiatry Clinical Practice 1998;2: 3-I7. 
6. Schmidtke, A., Bille-Brahe, U., De Leo, D., Kerkhof., A. Bjerke, T., Crepet, P., Haring, C., Hawton, K., Lonqvist, J., Michel, K., Pommereau, X., Querejeta, I., Phillipe, I., Salander-Renberg, E., Temesvary, B., Wasserman, D., Fricke, S., Weinacker, B., Sampaio-Faria, JG. Attempted suicide in Europe: rates, trends and sociodemographic characteristics of suicide attempters during the period 1989-1992. Results of the WHO/EURO Multicentre Study on Parasuicide. Acta Psychiatrica Scandinavica 1996; 93:327-338.

7. Aro, H., Marttunen, $\mathbf{M}$ and Lonqvist, J.Adolescente suicides in Finland. En 3rd. European Symposium .Suicidal Behaviour and Risk Factors (Ferrari, Bellini, Crepet .Editors) PP 7I-76. Monduzzi editori. Bologna 1990.

8. Davidson, L., Rosenberg, M., Mercy, J., Franklin, J., Simmons, J. An epidemiologic study of risk factors in two teenage suicide clusters. En 3rd. European Symposium. Suicide Behaviour and Risk Factors (Ferrari. Bellini, Crepet. Editors pp 505-514. Monduzzi Editori. Bologna.1990.

9. Platt, S y Kreitman, N. Parasuicide and unemployment among young men in Edinburgh 1968-1982. Psychological Medicine 1985; 15: II3-123.

10. Platt, S. Parasuicide and unemployment. British Journal of Psychiatry 1986; I49: 40I-405.

I I. Platt, S y Dyer, JAT. Psychological correlates of unemployment among male parasuicide in Edinburgh. British Journal of Psychiatry 1987; 151: 27-32.

12. Hawton, K., Fagg, J y Simkin, S. Female unemployment and attempted suicide. British Journal of Psychiatry 1988; 152: 632-637.

13. Moesler, TAM., Pontzen, W y Rummler, W. The relationship between suicdal acts and unemployment. En 3rd, European Symposium. Suicidal Behaviour and Risk Factors. (Ferrari, Bellini, Crepet. Editors). pp I89-194. Monduzzi Editori. Bologna. 1990.

14. Diekstra, RFW y Van Egmond, M. Suicide and attempt suicide in general practice 1976-1986. Acta Psychiatrica Scandinavica. 1989; 79: 268-279.

15. Kreitman, $\mathbf{N}$ y Casey, P. Repetition of parasuicide: an epidemiological and clinical study. British Journal of Psychiatry. 1988, 153: 792-800.

16. Hawton, K y Fagg, J. Repetition of attempted suicide: the performance of the Edinburgh predictive scales in patients in Oxford. Archives of Suicide Research. 1995, I: 26I-272.

17. Tuckman, J y Youngman, WF. A scale for assesing suicide risk of attempted suicides. Journal of Clinical Psychology. 1968, 24: 17-19. 